\title{
Docking study of flavonoid derivatives as potent inhibitors of influenza H1N1 virus neuraminidase
}

\author{
SEYED MAHDI SADATI ${ }^{1}$, NEMATOLLAH GHEIBI ${ }^{2}$, \\ SAEED RANJBAR $^{3}$ and MOHAMMAD SADEGH HASHEMZADEH ${ }^{1}$ \\ ${ }^{1}$ Applied Virology Research Center, Baqiyatallah University of Medical Sciences, Tehran 14359-16471; \\ ${ }^{2}$ Cellular and Molecular Research Center, Qazvin University of Medical Sciences, Qazvin 34156-13911; \\ ${ }^{3}$ Department of Medical Biotechnology, Faculty of Medical Sciences, Tarbiat Modares University, Tehran 14115-111, Iran
}

Received March 8, 2018; Accepted October 10, 2018

DOI: $10.3892 /$ br.2018.1173

\begin{abstract}
Influenza type A is considered as a severe public health concern. The mechanism of drugs applied for the control of this virus depends on two surface glycoproteins with antigenic properties, namely hemagglutinin (HA) and neuraminidase (NA). HA aids the virus to penetrate cells in the early stage of infection and NA is an enzyme with the ability to break glycoside bonds, which enables virion spread through the host cell membrane. Since NA contains a relatively preserved active site, it has been an important target in drug design. Oseltamivir is a common drug used for the treatment of influenza infections, for which cases of resistance have recently been reported, giving rise to health concerns. Flavonoids are natural polyphenolic compounds with potential blocking effects in the neuraminidase active site. Based on their antiviral effect, the flavonoids quercetin, catechin, naringenin, luteolin, hispidulin, vitexin, chrysin and kaempferol were selected in the present study and compared alongside oseltamivir on molecular docking, binding energy and active site structure, in order to provide insight on the potential of these compounds as targeted drugs for the control and treatment of influenza type A. The molecular characterization of flavonoids with binding affinity was performed using AutoDock Vina software. The results indicated that these compounds may effectively block the NA active site. Therefore, these natural compounds derived from fruits have the potential for development into drugs for controlling influenza, which may aid overcome the clinical challenge of the H1N1 strain epidemic.
\end{abstract}

Correspondence to: Dr Mohammad Sadegh Hashemzadeh, Applied Virology Research Center, Baqiyatallah University of Medical Sciences, Sheikh Bahaei Street, Tehran 14359-16471, Iran E-mail: dr_hashemzadeh@bmsu.ac.ir

Key words: influenza type A, neuraminidase, flavonoids, molecular docking

\section{Introduction}

Influenza type A virus is historically among the most aggressive virus' to have been identified, having caused several global epidemics. Between 1918 and 1919 this virus caused a mortality rate accounting for 5\% of the world population (1-3). Unfortunately, the potential hazard and spread of the virus remain to be considered as a threat to human health (4). Surface glycoproteins with antigenic properties including hemagglutinin (HA) and neuraminidase (NA) account for the emergence of novel subtypes of type A influenza and of novel forms of influenza. To date, 18 subtypes of $\mathrm{HA}(\mathrm{H} 1-\mathrm{H} 18)$ and 11 subtypes of NA (N1-N11) have been identified $(5,6)$. Hemagglutinin binds to sialic acid receptor for viral entry into the host cell (7). NA is an enzyme with the ability to break glycoside bonds, which may block cell-surface sialic acid receptor binding and allow virion spread across the host cell membrane. In the absence of this mechanism, virions accumulate on the cell membrane (8). In this manner NA may serve a facilitating role in the early stage of infection into endothelial cells (9).

Due to the importance of NA in virus distribution and its relatively preserved active site, it is an important target of drug design for control of influenza infections (10). Among previously developed drugs, oseltamivir, which targets NA, has been identified as the most effective, with an inhibitory effect on the N1, N2 and N9 subtypes $(11,12)$. However, several cases of resistance reported for this drug raises concern, since lack of disease control can be fatal with the potential for epidemic spread (3,13-15).

Flavonoids are naturally occurring, polyphenolic compounds of relatively low molecular weight that exhibit benzopyrone structure, produced as secondary metabolites in plants to stimulate growth during the state of tension $(16,17)$. Due to the reported antioxidant, antibacterial and antiviral effects, presence in normal daily diet and minimal side-effects of flavonoids, they are considered useful resources for drug design $(8,18-22)$. Initial reports on the use of flavonoids as pharmaceutical compounds emerged in the 1940s, when the potential of these compounds was evaluated in the prevention and treatment of coronary heart disease and for their anti-cancer, anti-inflammatory, antibacterial and antiviral 
effects (23-29). Due to the diversity of flavonoids, the use of computational designs and molecular information on flavonoid compounds enables the selection of the optimum compounds in investigations aimed at identifying alternative drugs with greater impact.

Compared with laboratory techniques, molecular data-based methods present the possibility to identify compounds with potent inhibitory effects, predict their structure and design effective drugs in a rapid and high-throughput approach. Compounds predicted through such computational methods require approval via classic experimental procedures (30-32). In fact, the study of molecular data offers the potential to select the most suitable compounds for treatment. Such a benefit is not possible without the need for a glossary of information, which would otherwise be time-consuming. In this regard, docking as the first medical computational design tool provides a method based on the prediction of binder energy interactions (using platforms including MetaPocket, GRAMM-X and HEX Protein Docking). The optimum orientation and interaction of the molecules with the lowest binding energy can be thus determined with the three-dimensional information index of the protein-protein binding (33-35).

Due to the abundance of research on the antiviral effects of flavonoids, in the present study, a molecular analysis of the binding affinity of various flavonoids was conducted by the docking method to determine the potential of these compounds as drug candidates for the control and treatment of influenza type A. The results indicated that the binding energy of the majority of flavonoids studied was high at the active site of neuraminidase N1 compared with oseltamivir, which may provide a basis for the replacement of oseltamivir with such flavonoids-based compounds.

\section{Materials and methods}

Molecular analysis protocol. Protein-ligand reactions, binding mode and binding affinity of neuraminidase N1 with oseltamivir and the selected flavonoids were determined via the docking method through the following steps:

Proteins. The Protein Data Bank (PDB) file of the region of neuraminidase N1 which interacts with oseltamivir (PDB code:3ti6) was obtained. Water molecules were removed and non-polar hydrogen molecules were added to the protein structure in Python molecule viewer setting of PyMOL v1.9 (http://www.pymol.org), which obtained the active site of the protein with a size of $24 \times 34 \times 22$ at $1 \AA$ grid spacing.

Ligands. CID files and molecular ligand data were obtained from PubChem on the NCBI database (https://pubchem. ncbi.nlm.nih.gov/search/search.cgi) (Table I). Ligands were converted to pdbqt format in Open Babel (https://openbabel. org/) GUI 2.3.2a setting.

Protein-ligand docking. Docking was performed in AutoDock Vina (http://vina.scripps.edu/), which predicts interactions between small molecules and proteins.

Determination of protein active site. The amino acids relevant to binding in the active site of the protein were determined using MetaPocket 2.0 (projects.biotec.tu-dresden. de/metapocket).

Analysis. Results were viewed and analyzed with PyMOL v1.9.

\section{Results}

Compound binding. The results indicated that all tested flavonoid compounds (Fig. 1) exhibited relatively high potential for binding to the active site of neuraminidase N1 (Table II). Notably their binding energy was higher in comparison to that of oseltamivir. It is noteworthy that the docking site of these compounds and oseltamivir were similar.

Compound docking. Results of compound docking in PyMol viewer revealed that all the tested flavonoid compounds have affinity for the active site of neuraminidase N1, similar to oseltamivir (Fig. 2).

Based on previous literature, several flavonoid compounds were initially selected (16). Compounds including caffeic acid and caffeine were also included in the computational investigation, but the obtained results were insignificant compared with the reported compounds, and only the docking results of the compounds with highest binding energy were reported (Table II). The obtained docking results confirmed the capability of the selected compounds in terms of effective molecular interaction with the investigated protein, when compared with oseltamivir; selected compounds were determined to have stronger affinities in binding with the active site of neuraminidase.

Protein active site. Amino acid residues involved in the interaction between the protein region and studied ligands were determined using MetaPocket 2.0 software (Fig. 3 and Table III).

Since the active site of neuraminidase N1, which interacts with oseltamivir, has a lower affinity than with the flavonoids, the selection of flavonoids in this study appeared successful. The selection of more optimal molecules exhibit a binding position with greater affinity may provide novel pharmaceutical approaches. In addition, the replacement of oseltamivir with flavonoids such as those currently tested when regarding the interaction with neuraminidase N1 may be a topic of interest, because of the importance of antimicrobials in the treatment of influenza type A, and flavonoids have been found to be effective against Gram-positive bacteria $(8,16)$.

\section{Discussion}

In recent years, among subtypes of influenza type A, H1N1 strain has been considered a challenge in terms of disease control and is a concern of national and international health organizations $(13,29)$. Several cases of mutations in the active site of neuraminidase have been reported, and may lead to resistance to oseltamivir, as a drug commonly administered for influenza $(14,15)$. These cases highlight the need for broader research aimed at determining alternative therapeutic methods.

The aim of the present study was to provide a basis for in vitro experiments from the perspective of protein-ligand molecular computing, for which AutoDock Vina software was used. Despite the rapidity of calculations, the quality of results obtained from AutoDock Vina software is comparable to those from AutoDock software $(31,32)$. Another aim was to shed light on the public notion regarding the potential medicinal effects of flavonoid consumption on influenza type A $(36,37)$. 
Table I. Molecular data of studied flavonoids and oseltamivir.

\begin{tabular}{lccccc}
\hline Compound & $\begin{array}{c}\text { Molecular } \\
\text { weight, g/mol }\end{array}$ & $\begin{array}{c}\text { Molecular } \\
\text { formula }\end{array}$ & & IUPAC name & Compound ID \\
\hline Oseltamivir & 312.4 & $\mathrm{C}_{16} \mathrm{H}_{28} \mathrm{~N}_{2} \mathrm{O}_{4}$ & ethyl (3R,4R,5S)-4-acetamido-5-amino-3-pentan-3-yloxycyclohe & 65028 \\
Quercetin & 302.2 & $\mathrm{C}_{15} \mathrm{H}_{10} \mathrm{O}_{7}$ & 2-(3,4-dihydroxyphenyl)-3,5,7-trihydroxychromen-4-one & 5280343 \\
Catechin & 290.3 & $\mathrm{C}_{15} \mathrm{H}_{14} \mathrm{O}_{6}$ & (2S,3R)-2-(3,4-dihydroxyphenyl)-3,4-dihydro-2H-chromene-3,5 & 73160 \\
Naringenin & 272.3 & $\mathrm{C}_{15} \mathrm{H}_{12} \mathrm{O}_{5}$ & 5,7-dihydroxy-2-(4-hydroxyphenyl)-2,3-dihydrochromen & 439246 \\
Luteolin & 286.2 & $\mathrm{C}_{15} \mathrm{H}_{10} \mathrm{O}_{6}$ & 2-(3,4-dihydroxyphenyl)-5,7-dihydroxychromen-4-one & 5280445 \\
Dinatin & 300.3 & $\mathrm{C}_{16} \mathrm{H}_{12} \mathrm{O}_{6}$ & 5,7-dihydroxy-2-(4-hydroxyphenyl)-6-methoxychromen-4-one & 5281628 \\
Vitexin & 432.4 & $\mathrm{C}_{21} \mathrm{H}_{20} \mathrm{O}_{10}$ & 5,7-dihydroxy-2-(4-hydroxyphenyl)-8-[(2S,3R,4R,5S,6R)-3,4,5 & 5280441 \\
Chrysin & 254.2 & $\mathrm{C}_{15} \mathrm{H}_{10} \mathrm{O}_{4}$ & 5,7-dihydroxy-2-phenylchromen-4-one & 5281607 \\
Kaempferol & 286.2 & $\mathrm{C}_{15} \mathrm{H}_{10} \mathrm{O}_{6}$ & 3,5,7-trihydroxy-2-(4-hydroxyphenyl)chromen-4-one & 5280863
\end{tabular}

IUPAC, International Union of Pure and Applied Chemistry.

Table II. Docking energy of selected flavonoid compounds and oseltamivir.

\begin{tabular}{lc}
\hline Compound & AutoDock energy, Kcal/mol \\
\hline Oseltamivir & -5.8 \\
Quercetin & -6.8 \\
Catechin & -7.2 \\
Naringenin & -6.9 \\
Luteolin & -7.1 \\
Dinatin & -6.8 \\
Vitexin & -7.5 \\
Chrysin & -6.8 \\
Kaempferol & -6.8
\end{tabular}

Flavonoids are currently identified not as medicine, but as necessary elements of daily diet that aid functioning of the immune system $(19,20)$. Due to their antioxidant activity, flavonoid compounds may regulate systemic anti-inflammatory reactions (16). Thus, applying them as a classical medicine does not appear a priority. Therefore, the present study aimed to highlight the overall effects of flavonoids as a dietary factor on the prevention, control and treatment of influenza $(38,39)$.

With the development of nanobiotechnological drug design, targeted delivery of flavonoid compounds to target tissues now appears a possibility (40). Via this method, the systemic side-effects of flavonoids during drug intake may be prevented and the therapeutic dose of flavonoids may be delivered directly to the targeted tissue, leading to improved efficiency of these drugs in lower doses.

Determining precisely the interactions in an active analogous position with antibiotics and candidate flavonoids may aid to better determine the alternatives. Since the variety of antibiotic sources useful for treatment is limited, and in recent decades there has been a lack of studies introducing novel antibiotics, the currently available antibiotics are becoming increasingly less effective due to the high rates of mutation<smiles>O=c1cc(-c2ccccc2)oc2cc(O)cc(O)c12</smiles>

Chrysin<smiles>O=c1c(O)c(-c2ccc(O)cc2)oc2cc(O)cc(O)c12</smiles>

Kaempferol<smiles>O=C1CC(c2ccc(O)cc2)Oc2cc(O)cc(O)c21</smiles>

Naringenin<smiles>O=c1cc(-c2ccc(O)cc2)oc2c(C3OC(CO)[C@@H](O)[C@H](O)[C@H]3O)c(O)cc(O)c12</smiles>

Vitexin<smiles>Oc1cc(O)c2c(c1)O[C@H](c1ccc(O)c(O)c1)C(O)C2</smiles>

Catechin<smiles>COc1c(O)cc2oc(-c3ccc(O)cc3)cc(=O)c2c1O</smiles><smiles>O=c1cc(-c2ccc(O)c(O)c2)oc2cc(O)cc(O)c12</smiles>

Luteolin<smiles>O=c1c(O)c(-c2ccc(O)c(O)c2)oc2cc(O)cc(O)c12</smiles>

Quercetin
Figure 1. Chemical formulas of the studied flavonoids and oseltamivir.

in viruses and bacteria (41-44). The performance of comprehensive studies to identify novel sources with the potential of replacing or at least complementing current antibiotics is considered a priority in the pharmaceutical industry. There are numerous antioxidant sources with antibiotic potential against viruses in nature, including fruits, vegetables and honey (45). So far, their established antioxidant effects have often been limited to applications in traditional medicine (46-49). Among these potentially antioxidant compounds are flavonoids; the 
Table III. Active site of neuraminidase N1 determined by MetaPocket 2.0.

\begin{tabular}{|c|c|c|c|c|c|}
\hline & Amino acid & Amino acid & Amino acid & Amino acid & Amino acid \\
\hline RESI & TYR_A"^207^" & ASN_A"^208^" & GLY_A"^209^" & ILE_A"^210^^" & ARG_A"^ $172^{\wedge "}$ \\
\hline RESI & PHE_A"^173^" & CYS_B"^129^" & PRO_B"^162^" & ILE_B"^163^" & GLU_B"^165^" \\
\hline RESI & GLU_B"^128^^" & LEU_B"^127^" & TYR_B"^100^" & GLU_A"^174^^" & LYS_A"^206^^" \\
\hline RESI & THR_A"^191^" & TRP_A"^189^" & SER_B"^101^^" & GLY_B"^164^" & LEU_B"^412^D" \\
\hline RESI & ARG_B"^130^" & PHE_A"^132^" & SER_A"^171^" & CYS_B"^124^" & GLN_B"^412^" \\
\hline RESI & GLU_A"^128^^" & SER_A"^125^" & ASN_A"^188^" & LEU_A"A $127^{\wedge "}$ & ARG_A"A $130^{\wedge "}$ \\
\hline RESI & HIS_A"^184^" & ILE_A"^187^" & SER_A"^82^" & PRO_A"^126^^" & GLY_A"^186^" \\
\hline RESI & VAL_A"^83^" & SER_A"^175^" & & & \\
\hline
\end{tabular}

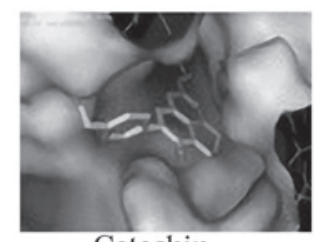

Catechin

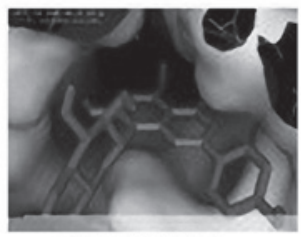

Kaempferol

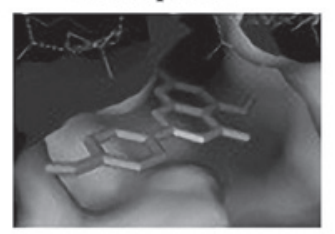

Naringenin

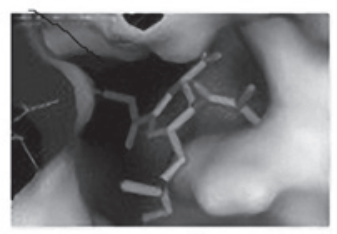

Quercetin

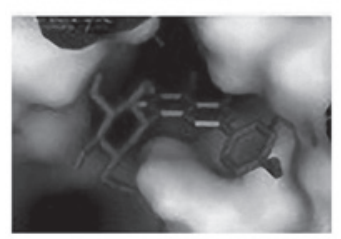

Vitexin

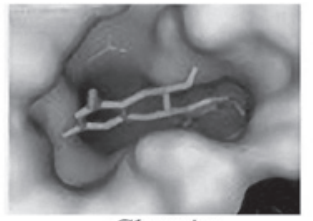

Chrysin

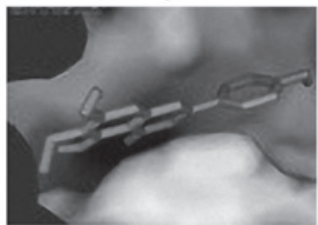

Hispidulin

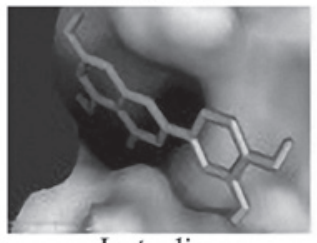

Luteolin

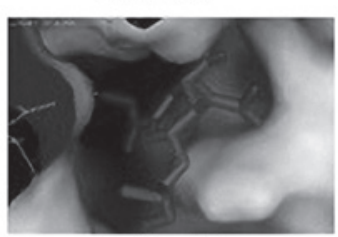

Oseltamivir

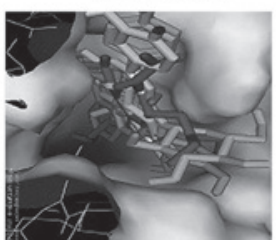

Complex
Figure 2. Docking results obtained with AutoDock Vina using PyMOL indicated that similar to oseltamivir, the selected flavonoids have affinity to bind with the activity site of neuraminidase (based on the results in Table II).

study of these compounds is thus warranted to select antibiotic candidates (50).

The establishment of novel drugs against influenza type A, for application when mutations in the binding site of the drug occur, appears imperative. Since the binding energies and active site interactions of compounds studied herein were comparable to oseltamivir, further evaluation is recommended

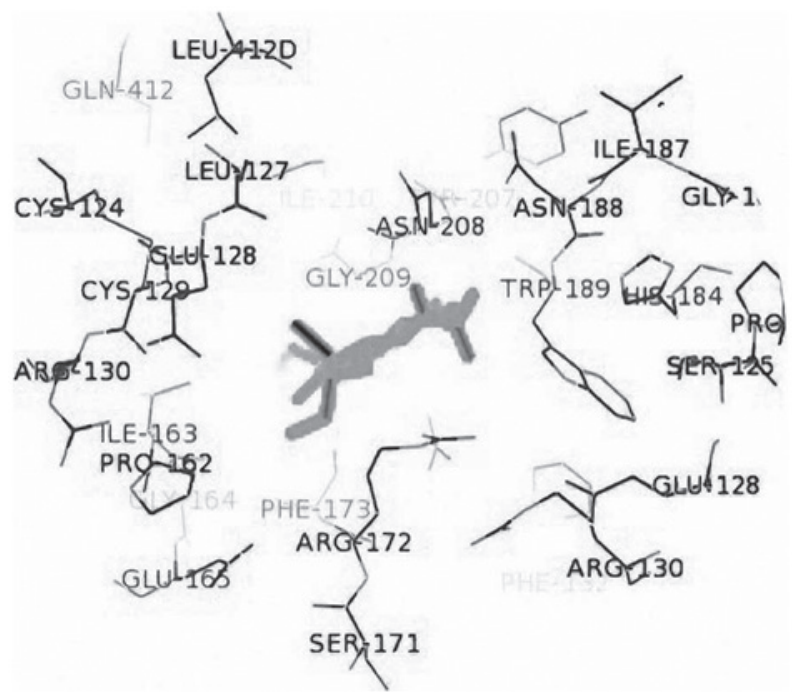

Figure 3. Active site of protein with oseltamivir determined using MetaPocket 2.0.

with the ultimate aim of introducing a novel generation of drugs in this field.

\section{Acknowledgements}

The authors thank Dr Ruhollah Dorostkar at Baqiyatallah University of Medical Sciences, Tehran, Iran, for providing suggestions on the article and servers used.

\section{Funding}

No funding was received.

\section{Availability of data and materials}

The datasets used and/or analyzed during the current study are available from the corresponding author on reasonable request.

\section{Authors' contributions}

SMS conceived and designed the study. NG analyzed the data. SR wrote the article. MSH performed the laboratory and server procedures. All authors read and approved the final manuscript. 


\section{Ethics approval and consent to participate}

Not applicable.

\section{Patient consent for publication}

Not applicable.

\section{Competing interests}

The authors declare that they have no competing interests.

\section{References}

1. Almond D: Is the 1918 Influenza pandemic over? Long-term effects of in utero Influenza exposure in the post-1940 US population. J Polit Econ 114: 672-712, 2006.

2. Taubenberger JK and Morens DM: 1918 Influenza: The mother of all pandemics. Emerg Infect Dis 12: 15-22, 2006.

3. Neumann G, Noda T and Kawaoka Y: Emergence and pandemic potential of swine-origin H1N1 influenza virus. Nature 459: 931-939, 2009.

4. Neumann G and Kawaoka Y: Transmission of influenza A viruses. Virology 479-480: 234-246, 2015.

5. Mallajosyula VV, Citron M, Ferrara F, Lu X, Callahan C, Heidecker GJ, Sarma SP, Flynn JA, Temperton NJ, Liang X, et al: Influenza hemagglutinin stem-fragment immunogen elicits broadly neutralizing antibodies and confers heterologous protection. Proc Natl Acad Sci USA 111: E2514-E2523, 2014.

6. Trott $\mathrm{O}$ and Olson AJ: AutoDock Vina: Improving the speed and accuracy of docking with a new scoring function, efficient optimization, and multithreading. J Comput Chem 31: 455-461, 2010

7. Weis W, Brown JH, Cusack S, Paulson JC, Skehel JJ and Wiley DC: Structure of the influenza virus haemagglutinin complexed with its receptor, sialic acid. Nature 333: 426-431, 1988.

8. Sibi G, Dhananjaya K and Bhimanagouda RP. Molecular docking and receptor based analysis of H1N1 antiviral drugs. Bioresearch Bull 2: 109-113, 2011.

9. Wang YT, Chan CH, Su ZY and Chen CL: Homology modeling, docking, and molecular dynamics reveal HR1039 as a potent inhibitor of $2009 \mathrm{~A}(\mathrm{H} 1 \mathrm{~N} 1)$ influenza neuraminidase. Biophys Chem 147: 74-80, 2010.

10. Seniya C, Khan GJ, Misra R, Vyas V and Kaushik S: In-silico modelling and identification of a possible inhibitor of H1N1 virus. Asian Pac J Trop Dis 4: S467-S476, 2014.

11. Govorkova EA, Ilyushina NA, McClaren JL, Naipospos TS Douangngeun B and Webster RG: Susceptibility of highly pathogenic H5N1 influenza viruses to the neuraminidase inhibitor oseltamivir differs in vitro and in a mouse model Antimicrob Agents Chemother 53: 3088-3096, 2009.

12. Park JW and Jo WH: Computational design of novel, high-affinity neuraminidase inhibitors for H5N1 avian influenza virus. Eur J Med Chem 45: 536-541, 2010.

13. Du QS, Wang SQ, Huang RB and Chou KC: Computational $3 \mathrm{D}$ structures of drug-targeting proteins in the 2009-H1N1 influenza A virus. Chem Phys Lett 485: 191-195, 2010.

14. Gupta CL, Akhtar S, Bajpaib P, Kandpal KN, Desai GS and Tiwari AK: Computational modeling and validation studies of 3-D structure of neuraminidase protein of H1N1 influenza A virus and subsequent in silico elucidation of piceid analogues as its potent inhibitors. EXCLI J 12: 215-225, 2013.

15. Bloom JD, Gong LI and Baltimore D: Permissive secondary mutations enable the evolution of influenza oseltamivir resistance. Science 328: 1272-1275, 2010

16. Kumar S and Pandey AK: Chemistry and biological activities of flavonoids: an overview. ScientificWorldJournal 2013: 162750 , 2013.

17. Dixon RA, Dey PM and Lamb CJ: Phytoalexins: Enzymology and molecular biology. Adv Enzymol Relat Areas Mol Biol 55: $1-136,1983$.

18. Cushnie TP and Lamb AJ: Antimicrobial activity of flavonoids. Int J Antimicrob Agents 26: 343-356, 2005.

19. Orhan DD, Ozçelik B, Özgen S and Ergun F: Antibacterial, antifungal, and antiviral activities of some flavonoids. Microbiol Res 165: 496-504, 2010
20. Cushnie TP and Lamb AJ: Recent advances in understanding the antibacterial properties of flavonoids. Int J Antimicrob Agents 38: 99-107, 2011.

21. Havsteen BH: The biochemistry and medical significance of the flavonoids. Pharmacol Ther 96: 67-202, 2002.

22. Middleton E Jr, Kandaswami C and Theoharides TC: The effects of plant flavonoids on mammalian cells: Implications for inflammation, heart disease, and cancer. Pharmacol Rev 52: 673-751, 2000.

23. Bandaruk Y, Mukai R and Terao J: Cellular uptake of quercetin and luteolin and their effects on monoamine oxidase- $\mathrm{A}$ in human neuroblastoma SH-SY5Y cells. Toxicol Rep 1: 639-649, 2014.

24. Li BQ, Fu T, Dongyan Y, Mikovits JA, Ruscetti FW and Wang JM: Flavonoid baicalin inhibits HIV-1 infection at the level of viral entry. Biochem Biophys Res Commun 276: 534-538, 2000.

25. Alam MA, Subhan N, Rahman MM, Uddin SJ, Reza HM and Sarker SD: Effect of citrus flavonoids, naringin and naringenin, on metabolic syndrome and their mechanisms of action. Adv Nutr 5: 404-417, 2014

26. Ganugapati J, Mukkavalli S and Sahithi A: Docking studies of green tea flavonoids as insulin mimetics. Int J Comput Appl 30: 48-52, 2011.

27. Jeong HJ, Ryu YB, Park S-J, Kim JH, Kwon H-J, Kim JH, Park $\mathrm{KH}$, Rho MC and Lee WS: Neuraminidase inhibitory activities of flavonols isolated from Rhodiola rosea roots and their in vitro anti-influenza viral activities. Bioorg Med Chem 17: 6816-6823, 2009.

28. Ganugapati J, Baldwa A and Lalani S: Molecular docking studies of banana flower flavonoids as insulin receptor tyrosine kinase activators as a cure for diabetes mellitus. Bioinformation 8 : 216-220, 2012.

29. Lu SJ and Chong FC: Combining molecular docking and molecular dynamics to predict the binding modes of flavonoid derivatives with the neuraminidase of the $2009 \mathrm{H} 1 \mathrm{~N} 1$ influenza A virus. Int J Mol Sci 13: 4496-4507, 2012.

30. Huang SY and Zou X: Advances and challenges in protein-ligand docking. Int J Mol Sci 11: 3016-3034, 2010.

31. Seeliger D and de Groot BL: Ligand docking and binding site analysis with PyMOL and Autodock/Vina. J Comput Aided Mol Des 24: 417-422, 2010.

32. Morris GM, Huey R and Olson AJ: Using AutoDock for ligand-receptor docking. Curr Protoc Bioinformatics 24: 8.14.1-8.14.40, 2008.

33. Ramharack P and Soliman MES: Bioinformatics-based tools in drug discovery: the cartography from single gene to integrative biological networks. Drug Discov Today 23: 1658-1665, 2018

34. Munsamy G and Soliman MES: Homology modeling in drug discovery-an update on the last decade. Lett Drug Des Discov 14: 1099-1111, 2017.

35. Tsujikawa H, Sato K, Wei C, Saad G, Sumikoshi K, Nakamura S, Terada T and Shimizu K: Development of a protein-ligand-binding site prediction method based on interaction energy and sequence conservation. J Struct Funct Genomics 17: 39-49, 2016.

36. Liu AL, Wang HD, Lee SM, Wang YT and Du GH: Structure-activity relationship of flavonoids as influenza virus neuraminidase inhibitors and their in vitro anti-viral activities. Bioorg Med Chem 16: 7141-7147, 2008.

37. Luria SE and Delbrück M: Mutations of bacteria from virus sensitivity to virus resistance. Genetics 28: 491-511, 1943.

38. Russo M, Spagnuolo C, Tedesco I, Bilotto S and Russo GL: The flavonoid quercetin in disease prevention and therapy: Facts and fancies. Biochem Pharmacol 83: 6-15, 2012.

39. Kuo SM: Dietary flavonoid and cancer prevention: Evidence and potential mechanism. Crit Rev Oncog 8: 47-69, 1997.

40. Chen Y, Wu Q, Song L, He T, Li Y, Li L, Su W, Liu L, Qian Z and Gong C: Polymeric micelles encapsulating fisetin improve the therapeutic effect in colon cancer. ACS Appl Mater Interfaces 7: 534-542, 2015.

41. Drake JW: The distribution of rates of spontaneous mutation over viruses, prokaryotes, and eukaryotes. Ann N Y Acad Sci 870 (1 MOLECULAR STR): 100-107, 1999.

42. Elena SF and Lenski RE: Evolution experiments with microorganisms: The dynamics and genetic bases of adaptation. Nat Rev Genet 4: 457-469, 2003.

43. Duffy S, Shackelton LA and Holmes EC: Rates of evolutionary change in viruses: Patterns and determinants. Nat Rev Genet 9: 267-276, 2008

44. Yogesh M and Saurabh S: Flavonoid: a pharmacologically significant scaffold. J Pharm Pharm Sci 6: 488-504, 2017. 
45. Eteraf-Oskouei T and Najafi M: Traditional and modern uses of natural honey in human diseases: A review. Iran J Basic Med Sci 16: 731-742, 2013.

46. Viuda-Martos M, Ruiz-Navajas Y, Fernández-López J and Pérez-Álvarez JA: Functional properties of honey, propolis, and royal jelly. J Food Sci 73: 117-124, 2008.

47. Gupta PD and Birdi TJ: Development of botanicals to combat antibiotic resistance. J Ayurveda Integr Med 8: 266-275, 2017.

48. Stanković N, Mihajilov-Krstev T, Zlatković B Stankov-Jovanović V, Mitić V, Jović J, Čomić L, Kocić B and Bernstein N: Antibacterial and antioxidant activity of traditional medicinal plants from the Balkan Peninsula. NJAS Wagening J Life Sci 78: 21-28, 2016.
49. Lee JH, Cho S, Paik HD, Choi CW, Nam KT, Hwang SG and Kim SK: Investigation on antibacterial and antioxidant activities, phenolic and flavonoid contents of some thai edible plants as an alternative for antibiotics. Asian-Australas J Anim Sci 27: 1461-1468, 2014.

50. Gonçalves E, Moreira C, Grosso P, Andrade B, Valentão P and Romano A: Phenolic profile, antioxidant activity and enzyme inhibitory activities of extracts from aromatic plants used in Mediterranean diet. J Food Sci Technol 54: 219-227, 2017. 